\title{
Apport bénéfique des traitements par photons pour la réalisation de composants III-V de bonne qualité
}

\author{
J. Flicstein, J. Mba, P. Ossart et J.M. Le Solliec \\ France Telecom, CNET, PAB, Laboratoire de Bagneux, 196 avenue H. Ravera, \\ 92225 Bagneux, France
}

Résumé : Le développement d'une nouvelle source d'irradiations à éclairs modulable en longueur d'onde, a ouvert la voie à de nouvelles techniques de dépôt activé par photons. Ces techniques exploitent la possibilité pour certaines sources de fournir des irradiations combinées en ultraviolet lointain, en rayonnement visible et en infrarouge dans la gamme 160-5000 nm. Un paramètre important est la durée de l'irradiation impulsionnelle (inférieure à 30 microsecondes). La puissance de la source que nous décrivons ici dans I'ultraviolet lointain permet de réaliser à la fois le nettoyage, le dépôt et le recuit des diélectriques à basse température $\left(<400{ }^{\circ} \mathrm{C}\right)$ sur $\mathrm{Si}$ et InP. Nous décrivons plus particulièrement la réalisation de couches minces de $\mathrm{SiO}_{\mathrm{X}} \mathrm{N}_{\mathrm{y}}$ peu hydrogénées et exemptes de radicaux $\mathrm{OH}$ et d'eau. Nous montrons que les qualités obtenues par cette technique sont intrinsèques aux mécanismes élémentaires des dépôts photolytiques.

\section{Introduction}

La réalisation de composants actifs micro- et optoélectroniques à base de semi-conducteurs III-V est motivée par les développements des réseaux de télécommunications optiques. Ces composants nécessitent la réalisation de couches minces diélectriques à base de silicium. Ces couches diélectriques ont un ensemble de propriétés physiques souhaitables afin de remplir toutes leurs fonctions optiques et électroniques[1,2]:

a. Transparence dans la bande des communications optiques $1,3<\lambda<1,55 \mu \mathrm{m}$,

b. Indice de réfraction uniforme et reproductible,

c. Epaisseur uniforme,

d. Interfaces et surfaces abruptes à faible rugosité microscopique,

e. Faible atténuation dans le domaine des hyperfréquences.

Les procédés de dépôt chimique en phase vapeur ( C V D ) pour la fabrication de couches diélectriques nécessitent typiquement des températures de substrat au-dessus de $700-800{ }^{\circ} \mathrm{C}$. Les matériaux III-V étant moins robustes aux traitements thermiques que le silicium, les procédés de fabrication à basse température sont naturellement privilégiés, par exemple par les techniques à base de plasma et les procédés photochimiques. Par ailleurs, des réactions de dépôt par photolyse indirecte faisant usage de mercure en tant que médiateur pour la photo dissociation du protoxyde d'azote en présence du silane, ont montré, en dehors du problème de la pollution par le mercuse, l'exisience ủes proùièmes d'adhérence et de stoechiométrie [3]. Egalement, plusieurs publications ont montré la possibilité d'utiliser la photo-dissociation par une source à raie unique continue en ultra violet profond ( VUV) comme technique de dépôt à basse température [4,5-7]. Par rapport aux autres sources de photons, utilisees par exemple en photo thermie rapide (RT CVD), l'irradiation par VUV présente au moins trois avantages. Premièrement, l'ensemble substrat /diélectrique "voit" des températures beaucoup plus réduites. Le bénéfice immédiat est la 
quasi-absence des dommages mécaniques et optiques dus à la contrainte thermique. Deuxièmement, le VUV contribue au contrôle de l'oxydation, jusqu'à l'oxydation complète de la couche đéposée [8]. Il a été montré que l'exposition de la silice partiellement oxydée à l'irradiation UV produit son oxydation totale [ 8,9 ]. Troisièmement, l'exposition du substrat aux VUV avant dépôt élimine la contamination de surface $[3,9]$.

Ce travail met en avant les avantages d'une nouvelle technique de dépôt dans laquelle on irradie les précurseurs par des tubes à éclairs ayant un spectre large bande de l'infrarouge $(5000 \mathrm{~nm})$ au VUV $(160 \mathrm{~nm})$.

Nous présenterons tout d'abord succintement les propriétes diélectriques des matériaux amorphes utilisés en micro et en optoélectronique. Dans la seconde partie nous détaillerons les réalisations des oxynitrures de silicium soit à composition stoechiométrique soit à composition variable obtenues en distinguant deux modes de fonctionnement de la lampe à éclairs. Nous montrerons que par ailleurs la luminance dans la gamme VUV et la distribution en longueurs d'onde d'une telle lampe peuvent être modulées et dépendent directement du courant appliqué dans le circuit extérieur.

Quelle que soit l'origine microscopique du mécanisme de dépôt de diélectrique et de nettoyage de surfaces, nous nous interrogerons enfin sur ses conséquences quant à la morphologie, la microstructure et en relation avec ses propriétés optiques pour les applications du matériau à insérer dans le futur composant.

\subsection{Optimisation de la constante diélectrique}

L'abaissement de la constante diélectrique est, entre autres, un but recherché pour que le diélectrique soit le plus adapté pour l'optique, ou comme couche diélectrique intermédiaire en micro-électronique. La valeur attendue de cette constante diélectrique dépendra de la nature et de la quantité de liaisons chimiques constituant la structure du matériau. Pour un matériau homogène, la valeur de la constante diélectrique peut être évalué grosso modo à l'aide du champ local de l'équation de Clausius-Mossotti [10]:

$$
\frac{\varepsilon-1}{\varepsilon+2} \frac{M}{\rho}=\frac{4 \pi}{3} N \alpha
$$

$M$ étant le poids moléculaire du matériau $(\mathrm{g} / \mathrm{mol}), \rho$ sa densité $\left(\mathrm{g} / \mathrm{cm}^{3}\right), N$ le nombre d'Avogadro et $\alpha$, la polarisabilité (le pouvoir de polarisation) du premier ordre. L'équation de Clausius-Mossotti n'est valable que pour les cristaux du système cubique. Pour les diélectriques amorphes, nous nous plaçons dans le cas isotrope. Prenons l'exemple de l'oxynitrure de silicium. On peut l'assimiler à un milieu constitué de $\mathrm{SiO}_{2}$ (a) et $\mathrm{Si}_{3} \mathrm{~N}_{4}$ (b) Les constituants sont mélangés de façon aléatoire:

$$
\frac{\varepsilon-1}{\varepsilon+2}=\frac{4 \pi}{3}\left(n_{a} \alpha_{i}+n_{b} \alpha_{b}\right)
$$

ou $\mathbf{n}$ est la densité volumique du constituant et $\varepsilon$ est la constante diélectrique effective de l'oxynitrure de silicium. En explicitant la constante diélectrique effective en fonction des constantes diélectriques de constituants, on obtient : 


$$
\frac{\varepsilon-1}{\varepsilon+2}=f_{x} \frac{\varepsilon x-1}{\varepsilon x+2}+f_{y} \frac{\varepsilon y-1}{\varepsilon y+2}
$$

De point de vue pratique il est plus commode d'utiliser : 1) non pas la valeur $\alpha$ elle-même, mais son multiple, valeur qui porte le nom de la polarisation moléculaire $P$ du diélectrique et 2) la réfraction molaire, $\mathrm{R}\left(\mathrm{cm}^{3} / \mathrm{mol}\right)$, selon Lorentz-Lorentz, qui permet l'additivité des polarisabilités et celle des volumes molaires $\left(V_{\text {mol }}=M / \rho\right)$ des éléments structuraux. du matériau [10]:

$$
R=\frac{n^{2}-1}{n^{2}+2} \frac{M}{\rho}
$$

La réfraction molaire est donc reliée à la structure moléculaire, l'indice de réfraction (n) et à la densité du diélectrique $\left(\mathrm{g} / \mathrm{cm}^{3}\right)$. De telle manière $\mathrm{R}$ peut être calculé pour un composé à partir des données expérimentales optiques, l'indice de réfraction et la densité. A partir du principe de l'additivité, et avec ( 4-1.1), $\mathrm{R}$ est décomposé et assigné aux différents éléments structuraux, pour un autre composé de structure voisine, et converti, pour estimer la valeur de la fonction diélectrique, $\varepsilon(v) \cong n^{2}(v)$. Pour les matériaux optiques à faible atténuation, la fonction diélectrique est peu dépendante de la fréquence $v$. Pour la prédiction du changement de la constante diélectrique de $\mathrm{SiO}_{\mathrm{x}} \mathrm{N}_{\mathrm{y}}$ quels que soient $\mathrm{x}$ et $\mathrm{y}$ :

$$
\varepsilon \cong \frac{1+\frac{2 R}{V m o l}}{1-\frac{R}{V m o l}}
$$

Ainsi à polarisabilité constante, l'éq. (5-1.1) montre la possibilité de diminuer la constante diélectrique, par des remplacements moléculaires qui augmentent le $\mathrm{V}_{\mathrm{mol}}$. Par exemple, pour générer $\mathrm{SiO}_{\mathrm{X}} \mathrm{N}_{\mathrm{y}}$ à partir de $\mathrm{Si}_{3} \mathrm{~N}_{4}$ et $\mathrm{SiO}_{2}$, suite à un échange de l'oxygène par azote et à l'augmentation de la proportion de celui ci par rapport à l'oxygène, en examinant l'éq. (5-1.1) on observe que, par rapport à $\mathrm{Si}_{3} \mathrm{~N}_{4}$, la constante diélectrique de $\mathrm{SiO}_{\mathrm{X}} \mathrm{N}_{\mathrm{y}}$ aura la tendance de diminuer.

\section{Description de la technique de dépôt VUV induit par une lampe à éclairs VUV-VIS- IR}

Une nouvelle technique basse température a été utilisée qui est une combinaison de dépôt CVD induit par irradiation en UV profond (VUV), concomitant avec l'irradiation dans le visible (VIS) et en infra-rouge (IR). Le réacteur de photolyse VUV-VIS-IR est équipé à l'extérieur d'une lampe contenant quatre tubes à éclairs et un réflecteur plan. Un jet d'azote chasse l'air et refroidit le tube. Typiquement, le tube à éclairs est linéaire et fonctionne par décharges électriques périodiques, pour une fréquence de répétition de 1 à $20 \mathrm{~Hz}$ de durée $\sim 30 \mu$ s. Le tube est en verre Suprasil et comporte deux électrodes ayant un écartement de $12 \mathrm{~cm}$. Il est rempli avec du krypton ou de l'argon. 
Le réacteur horizontal est une version modifiée du réacteur de dépôt $\mathrm{C} \mathrm{V} \mathrm{D} \mathrm{par} \mathrm{thermie}$ rapide ( $R$ T ) décrit précédemment [11]. Le réacteur construit par JIPELEC, JetLight 100 accepte des substrats de grande surface. Ceci concerne en particulier l'uniformité de dépôt grande surface. Les expériences sont effectuées en régime d'écoulement régulé en débit de chaque précurseur, la pression totale dans le réacteur etant régulée séparément. La direction de propagation de l'irradiation est normale à la surface du substrat.

\section{Obtention de matériaux optiques en couches minces et conditions expérimentales}

Les caractéristiques souhaitables pour la validation d'une technique de dépôt basse température pour la fabrication de multicouches de diélectriques doivent comprendre :

1) La capacité de produire des couches stoechiométriques et aussi des couches à stoechiométrie contrôlée.

2) Le taux de dépôt suffisamment élevé.

3) La capacité à produire un dépôt conforme à pente positive sur des substrats de morphologies variables.

4) La capacité à produire des couches uniformes sur des grandes surfaces.

5) La capacité à produire une large gamme d'épaisseurs.

Cette méthode basée sur la technique de dépôt VUV induit par une lampe à éclairs VUVVIS-IR produit des résultats très satisfaisants en ce qui concerne les quatre premiéres caractéristiques demandées $[1,12]$. Des résultats récents ont montré qu'un effort concentré sur le point 5) permettra de résoudre les difficultés rencontrées dans l'obtention des couches de diélectriques épaisses [13].

Les réactions photolytiques de dépôt ont été réalisées dans le réacteur FVUV CVD à basse pression, 0,42 mTorrs. L'étalonnage du flux de photons VUV et les caractéristiques spectrales du tube à éclairs ont été mesurées [12]. Les gaz précurseurs, silane, protoxyde d'azote et ammoniac, sont mélangés avant l'admission dans le réacteur. Les débits utilisés sont précisés dans la fig. 1. Le substrat est placé face à la lampe, à une distance de $75 \mathrm{~mm}$. Le chauffage est réglé par le taux de répétition des éclairs $(10$ et $20 \mathrm{~Hz})$. La température du dépôt a été maintenue constante durant une expérience ( $300-407^{\circ} \mathrm{C}$ ). Le substrat à été exposé au flux de précurseurs pendant 5 min avant l'allumage de la lampe.

Les spectres d'absorption en infrarouge ont été réalisés avec des dépôts sur substrats de silicium peu absorbants. L'appareil utilisé est un spectrophotomètre Nicolet $740 \mathrm{SX}$ à transformée de Fourier dans la gamme entre 400 et $4000 \mathrm{~cm}^{-1}$ avec un pas de $2 \mathrm{~cm}^{-1}$. Pour les études de morphologie de surface un AFM (Microscope à Force atomique ) Nanoscope III (Digital Instruments ) a été utilisé. Pour étudier l'évolution de la couche dans le temps du dépôt nous considérons les résultats de la mesure d'indice de réfraction. Les mesures d'indice de réfraction ont été effectuées (Rudolph Research / Auto El) à une longueur d'onde (laser He-Ne).

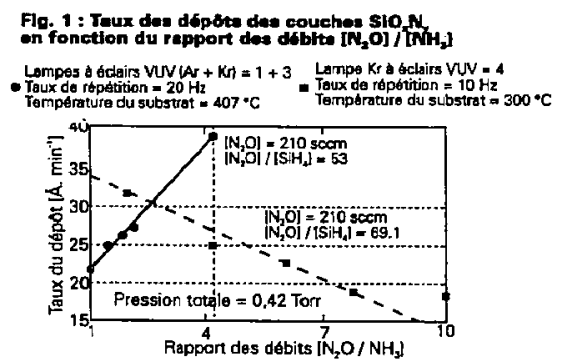




\section{Résultats et discussions}

La première condition nécessaire pour la réussite de la photolyse est la concordance entre les spectres d'absorption et le spectre d'émission de la lampe. Avec le spectre d'ammoniac, la limite supérieure de l'absorption se situe à $206 \mathrm{~nm}$. En revanche, la limite d'absorption est inférieure pour le protoxyde d'azote, en se situant à $185 \mathrm{~nm}$ seulement. Compte-tenu du spectre d'émission du tube argon à éclairs, le choix du protoxyde d'azote et de l'ammoniac, comme contributeurs de l'azote, présente un avantage important relatif à l'usage unique du protoxyde d'azote dans le mélange des précurseurs [ 14 ], contrairement à ce qui se passe, dans la décomposition du précurseur par pyrolyse optique, par exemple dans le cas de la photo-thermie rapide. Cette limite d'absorption du protoxyde d'azote est trop basse et donc ne convient pas au processus de photolyse induit par la lampe krypton à éclairs. Toutefois la lampe à $\mathbf{K r}$ est utile en dépit de cette limitation, l'intérêt étant la possibilité des couches $\mathrm{SiO}_{\mathrm{X}} \mathrm{N}_{\mathrm{y}}$ avec concentrations en $\mathrm{x}$ et y variables. Donc pour obtenir $\mathrm{SiO}_{\mathbf{X}} \mathrm{N}_{\mathbf{y}}$, la photolyse à été obtenue avec une source d'irradiation constituée de 4 tubes à éclairs remplis krypton. Dans une grande plage de rapport de débits, il est possible de réaliser des couches homogènes en composition avec un taux de dépôt décroissant, malgré l'augmentation du débit de protoxyde d'azote (voir fig. 1). On verra par la suite que l'indice de réfraction de telles couches reste contrôlable. Les remarques précédentes ne nous permettent évidement pas d'affirmer que les dépôts sont constitués des espèces provenant uniquement de la photolyse. Une méthode d'analyse élémentaire en utilisant la microscopie d'électrons Auger (A E S ) nous permet de vérifier cette hypothèse. La provenance dans le spectre Auger de l'oxygène et de l'azote est sans doute de source photolytique (fig. 2) à partir des précurseurs $\mathrm{N}_{2} \mathrm{O}$ et $\mathrm{NH}_{3}$. Le profil en profondeur obtenu montre l'evolution des concentrations atomiques dans la couche. La provenance du silicium dans le dépôt ne peut être que de nature indirecte, par la réaction de produits de photolyse avec le silane. Une analyse plus détaillée du mécanisme de la photolyse, qui génère les espèces constituantes du dépôt, à été abordée précédemment [15]. Donc, en associant des tubes différents à éclairs ( $\mathrm{Ar}$ et $\mathrm{Kr}$ ) ces résultats indiquent bien la réalisation d'un dépôt quasi-stoechiométrique de $\mathrm{Si}_{2} \mathrm{~N}_{2} \mathrm{O}$ avec une région de transition abrupte $(\sim 70 \dot{\mathrm{A}})$. Les propriétés structurales des couches ont été caractérisées par leurs spectres de vibrations. Les spectres FTIR obtenus ont mis en évidence que l'azote est incorporé dans la structure. La position en fréquence du mode d'élongation de la liaison $\mathrm{Si}-\mathrm{O}(\mathrm{N})$ est à $920 \mathrm{~cm}^{-1}$. Dans les limites de résolution de l'appareil, cette valeur du pic s'est montrée insensible au changement d'épaisseur [16]. Les couches comportent très peu de liaisons hydrogène : le mode d'élongation de la liaison $\mathrm{Si}_{2}-\mathrm{N}-\mathrm{H}$ est à $3373 \mathrm{~cm}^{-1}[15,17]$, Si-H étant absent à $880 \mathrm{~cm}^{-1}$ ( mode de torsion) et $2260 \mathrm{~cm}^{-1}$ (mode élongation).
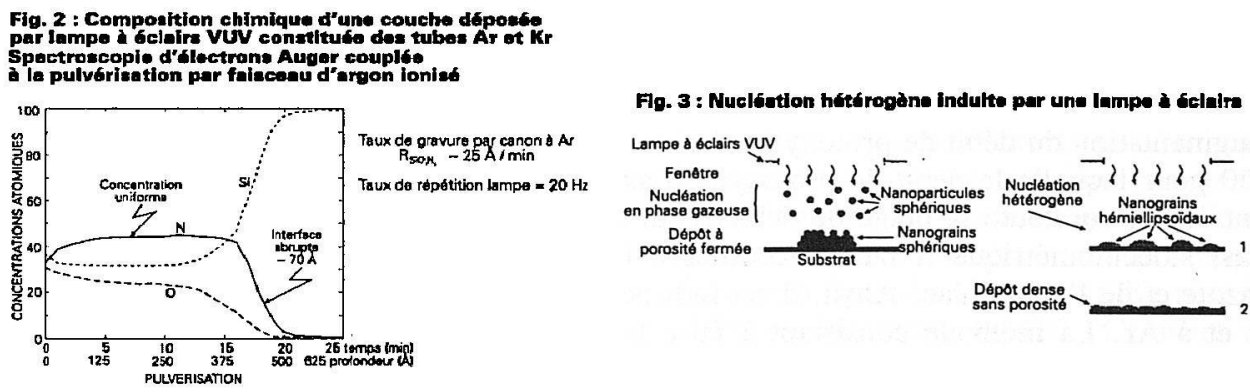
On peut supposer que ces résultats sont dus à la contribution IR, du fait de la relaxation et de la desorption par irradiation en infrarouge des fragments issus de la photolyse [18]. Malgré la faible température, nous notons une remarquable absence du dépôt des modes d'élongation et torsion pour $\mathrm{O}-\mathrm{H}$ et $\mathrm{H}_{2} \mathrm{O}\left(3200-3600 \mathrm{~cm}^{-1}\right)$ et respectivement $830-920 \mathrm{~cm}^{-1}$.

\subsection{Morphologie , microstructure et conséquences sur les propriétés optiques}

Un schéma de l'interaction typique entre l'irradiation VUV émise par le tube à éclairs et le précurseur, qui induit la photolyse du précurseur, permettra l'analyse qualitative du déroulement des premiers stades de la photonucléation en fonction de la luminance dans les trois régions différentes définies de manière arbitraire dans le réacteur (fig. 3):

I. La surface de la fenêtre et sa proximité,

II. Le volume du réacteur,

III. La surface du substrat et sa proximité.

Puisque la luminance est inversement proportionnelle au carré de la distance au tube à éclairs, la photonucléation sur le substrat s'explique par un meilleur coefficient de collage et la création de centres de nucléation induits par l'irradiation VUV. La variation de luminance entraîne des probabilités différentes de nucléation hétérogene, pour un coefficient de collage similaire, sur le substrat et la fenêtre du réacteur. Par contre, la nucléation hétérogene est prioritaire à la surface du substrat, où sont créés les centres actifs de charge (ACC). L'activité accrue des ACC est induite par l'irradiation VUV. Leur densité augmente avec la luminance sur le substrat, suite au changement induit du nombre de paires électron-trou. Pour une discussion plus détaillée on peut consulter $[15,18,19]$. Les couches obtenues n'ont pas de microstructure granulaire poreuse. Cette qualité est due à l'usage extensif des principes de la photonucléation hétérogène dirigée prioritairement vers le substrat. La nucléation hétérogene, sur les ACC présents, entraîne la constitution d'un dépôt plus dense qu'en leur absence (fig. 5). Toutefois, l'existence des ACC ne modifie apparemment pas la morphologie de la surface du dépôt. Une vue de la surface a été prise par un Microscope à Force Atomique. La fig.4 présente un résultat typique des morphologies. Ces formes régulières qui bénéficient d'une faible rugosité $(8 \AA$ ) peuvent être en accord avec un début de mode de croissance de type Volmer-Weber, ainsi que des simulations Monte Carlo (fig.5) l'ont confirmé [15]. Par ailleurs, une autre qualité morphologique intrinsèque, le recouvrement conforme des marches à pente positive par le dépôt, est favorisée pour le dépôt par photolyse; ceci par l'amélioration de l'adhésion dû au caractère physico-chimique de la technique de dépôt [3].

Concernant les propriétés optiques, l'uniformité des couches a été vérifiés systématiquement en plusieurs points. Les valeurs ainsi obtenues d'indice de réfraction ont été contrôlées par ellipsométrie spectroscopiques (SOPRA ES4G). Pour le même domaine de débits, la trace de photolyse partielle subsiste pour la lampe krypton à éclairs. Malgré l'augmentation du débit de protoxyde d'azote, l'indice de réfraction evolue de 1,58 jusqu'à 1,50 pour laquelle le débit de protoxyde d'azote est maximal. L'indice de réfraction reste contrôlable sur toute la plage de débits.Pour réaliser des couches d'oxynitrure de silicium quasi stoechiométrique il faut s'accorder à la fois au spectre d'absorption de protoxyde d'azote et de l'ammoniac. Ainsi, il est indispensable d'utiliser à la fois les tubes à éclairs à $\mathrm{Kr}$ et à Ar. La méthode consistant à faire la photolyse avec deux sources d'irradiations différentes est générale et peut être appliquée en réalisant une source associant différents 
tubes. La grande souplesse d'emploi d'un tel système est illustrée par les résultats obtenus (figs. 1,6 ). La valeur mesurée du taux de dépôt est proche de $40 \AA$ A $/ \mathrm{min}$ et les couches obtenues ont un indice de réfraction de $\sim 1,69$. Ces résultats sont encourageants puisque les techniques photochimiques ont la réputation de produire de matériaux moins denses. L'indice de réfraction de l'oxynitrure de silicium déposée par RTCVD à haute température $\left(\sim 750{ }^{\circ} \mathrm{C}\right)$ se situe à 1.70 [17]. Ce résultat constitue donc une première confirmation de notre modèle pour le dépôt induit par photons VUV basé sur la création des centres ACC [18].
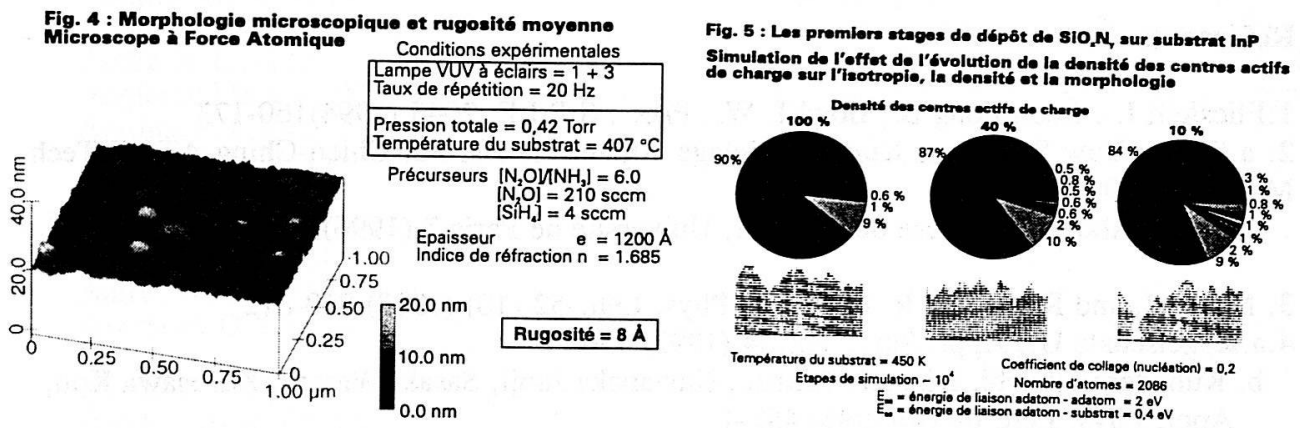

Fig. 6 : Evolution de I'indice de réfraction en fonction du rapport des débits $\left[\mathrm{N}_{2} \mathrm{O}\right] /\left[\mathrm{NH}_{3}\right]$

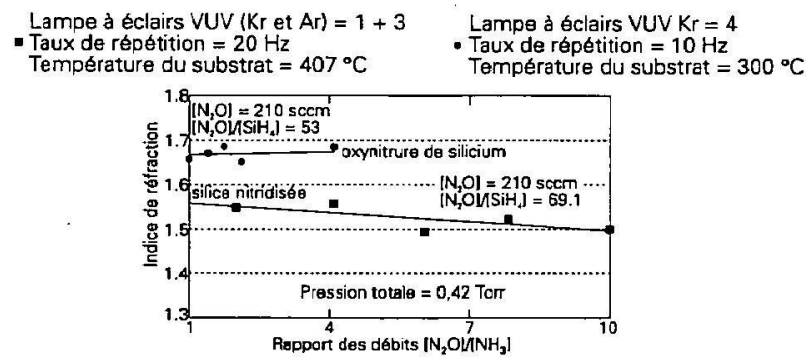

\section{Conclusions}

Une nouvelle approche de la photolyse à été développée pour réaliser des diélectriques de qualité micro-électronique. Elle consiste en l'utilisation sélective, localisée et concomitante d'irradiations incohérentes en ultraviolet profond et en proche infrarouge lors des dépots. Nous avons limité notre approche à la réalisątion de couches diélectriques sur les matériaux III-V et Si. Les effets mis en jeu ne sont pas encore totalement compris mais d'ores et déjà la versatilité des possibilités des dépóts de $\mathrm{SiO}_{\mathrm{x}} \mathrm{N}_{\mathrm{y}}$ est très intéressante au niveau de propriétés diélectriques obtenues. Les possibilités ont été démontrées en utilisant des mélanges à trois précurseurs : silane, protoxyde d'azote et ammoniac. Le procédé a associé pour la première fois dans une même configuration des tubes à éclairs à $\mathrm{Kr}$ et à $\mathrm{Ar}$ aboutissant un procédé simple d'emploi, réalisé à basse température $\left(<400{ }^{\circ} \mathrm{C}\right)$ ayant un budget thermique faible, et pour l'obtention de diélectriques amorphes de qualité microoptoélectronique. 


\section{Remerciements}

A M. O. Dulac pour les modifications apportées au bâti Jipelec, M. B. Lescaut pour l'aide expérimentale, Dr. E. Toussaere pour les mesures d'ellipsometrie spectroscopique, M. C. David du Laboratoire L2M/ CNRS pour les mesures par AFM et Dr J. F. Palmier pour discussions fructueuses.

\section{Références}

1.Flicstein J. , Leon Fong B., Boyd I. W., Proc . S P I E 2045,(1994)160-173

2. a.Obeng Yaw S.,Steiner Kurt G., Velaga Ankineedu N., Pal Chien-Ching,AT\&T Tech. May/June (1994)94

b.Sayah Abdeljalil, Thèse de doctorat, Université de Paris 7,(1996)

3. Marks J. and Robertson R. E., Appl. Phys. Lett.,52,(10) (1988) 810-812

4.a.Kogelschatz U., Appl. Surf. Sci.,54 (1992) 410

b. Kubodera Shoichi, Kitahara Mitsuo, Kawanaka Junji, Sasaki Wataru, Kurosawa Kou, Appl. Phys. Lett. 69 (4)(1996) 452-4

5. Bergonzo P. Ph. D. Thesis, University College London (1994)

6. Parada Garcia E. D. Sc. Thesis, Univ. Vigo, (Spain, 1995)

7. Bergonzo P. , Patel P. ,Boyd I. W. ,54,(1992) 424

8. Bradford A.P., Haas G., McFarland M. and Ritter E., Appl. Opt.,4 (1965) 971

9. Flicstein J., NissimY. I., Licoppe C., Vitel Y., French patent 9103964 (1991)

10. Urban A. ,Thèse de Docteur-Ingenieur, Université de Paris 6 (1983)

11. Nissim Y. I., Moison J. M., Houzay F., Lebland F., Licoppe C., and Bensoussan M., Appl. Surf. Sci. 46 (1990) 175

12. Flicstein J., Vitel Y., Dulac O., Debauche C., Nissim Y. I., Licoppe C.,Appl. Surf. Sci. 86 (1995)286-293

13. Flicstein J. et Mba J. Résultats non-publiés.

14. Debauche C. ,Thèse de doctorat, Université de Paris 6, (1994)

15. Flicstein J., Mba J, Le Solliec J.-M., Palmier J. F. Proc. SPIE ( soumis pour publication, 1996)

16. Devine R. A. B. J. Vac. Sci., Technol., A6, 3154 (1988)

17. Lebland F., Thèse de doctorat, Université de Paris 7, (1992)

18. Flicstein J.,Le Solliec J.-M. ( à soumettre pour publication)

19. Flicstein J. and Bourée J. E. , "Promoting photonucleation on semiconductor substrates for metallisation" in ,Photochemical processing of electronic materials, (Academic Press ,London, 1992) Boyd I. W. and Jackman R. B. Eds. , pp. 105-142 